\title{
Counting eigenvalues via the Krein signature in infinite-dimensional Hamiltonian systems
}

\author{
Todd Kapitula* \\ Department of Mathematics and Statistics \\ University of New Mexico \\ Albuquerque, NM 87131 \\ Panayotis G. Kevrekidis ${ }^{\dagger}$ \\ Björn Sandstede f $^{\ddagger}$ \\ Department of Mathematics and Statistics \\ University of Massachusetts \\ Department of Mathematics \\ Amherst, MA 01003 \\ Ohio State University \\ Columbus, OH 43210
}

March 15, 2004

\begin{abstract}
Spectra of nonlinear waves in infinite-dimensional Hamiltonian systems are investigated. We establish a connection via the Krein signature between the number of negative directions of the second variation of the energy and the number of potentially unstable eigenvalues of the linearization about a nonlinear wave. We apply our results to determine the effect of symmetry-breaking on the spectral stability of nonlinear waves in weakly coupled nonlinear Schrödinger equations.
\end{abstract}

\footnotetext{
*E-mail: kapitula@math.unm.edu

$\dagger$ E-mail: kevrekid@math.umass.edu

${ }_{\ddagger}^{\ddagger}$ E-mail: sandstede.1@osu.edu
} 


\section{Introduction}

We are interested in certain spectral-stability aspects of waves in Hamiltonian nonlinear partial differential equations (PDEs). The abstract framework that we shall work with consists of a Hamiltonian system

$$
u_{t}=J E^{\prime}(u), \quad u(0)=u_{0}
$$

on a Hilbert space $X$, where $J: X \rightarrow X$ is invertible and skew-symmetric, and $E: X \rightarrow \mathbb{R}$ is a $\mathcal{C}^{2}$-functional. We assume that the energy functional $E$ is invariant under the action of an abelian Lie group $G$ with Lie algebra $\mathfrak{g}$ so that (1.1) is equivariant under $G$. Solutions of particular interest are relative equilibria $\phi_{0}$ whose time evolution takes place on the group orbit $G \phi_{0}=\left\{g \phi_{0} ; g \in G\right\}$ so that $\phi(t) \in G \phi_{0}$ for all $t$. In fact, we have $\phi(t)=\exp (\omega t) \phi_{0}$ for an appropriate element $\omega \in \mathfrak{g}$, and the relative equilibrium $\phi_{0}$ is a genuine equilibrium of

$$
u_{t}=J\left[E^{\prime}(u)-J^{-1} T_{\omega} u\right]
$$

where $T_{\omega}$ is the generator of $\exp (\omega t)$. Spectral stability of the relative equilibrium $\phi_{0}$ is therefore determined by the spectrum of the linearization $J \mathcal{L}$ of (1.2) about $\phi_{0}$, where the self-adjoint operator $\mathcal{L}=E^{\prime \prime}\left(\phi_{0}\right)-J^{-1} T_{\omega}$ is the Hessian of the energy calculated in the frame $u \mapsto \exp (\omega t) u$ that moves along group orbit.

To associate a concrete example with this abstract framework, consider the system

$$
\partial_{t} q_{j}=\mathrm{i}\left[d_{j} \partial_{x x} q_{j}+\frac{\partial g}{\partial\left|q_{j}\right|^{2}}\left(\left|q_{1}\right|^{2}, \ldots,\left|q_{N}\right|^{2}\right) q_{j}\right], \quad x \in \mathbb{R}, \quad j=1, \ldots, N
$$

of coupled nonlinear Schrödinger equations (NLS) which is of the form (1.1) with $X=H^{1}\left(\mathbb{R}, \mathbb{C}^{N}\right), J=\mathrm{i}$ and

$$
E(q)=\int_{\mathbb{R}}\left[\sum_{j=1}^{N} d_{j}\left|\partial_{x} q_{j}\right|^{2}-g\left(\left|q_{1}\right|^{2}, \ldots,\left|q_{N}\right|^{2}\right)\right] \mathrm{d} x .
$$

Equation (1.3) is equivariant under the group $G=\mathbb{R} \times \mathbb{T}^{N}$, where $\mathbb{T}^{N}$ denotes the $n$-dimensional torus, which acts by translation in $x$ and by the rotations $q=\left(q_{j}\right) \mapsto \mathrm{e}^{\mathrm{i} \alpha} q=\left(\mathrm{e}^{\mathrm{i} \alpha_{j}} q_{j}\right)$ for $\alpha=\left(\alpha_{j}\right) \in \mathbb{T}^{N}$. In particular, each solution of the form $q(x, t)=\mathrm{e}^{\mathrm{i} \omega t} \phi_{0}(x)$ is a relative equilibrium of (1.3). Note that $T_{\omega}=\mathrm{i} \omega$ so that $\mathcal{L}=E^{\prime \prime}\left(\phi_{0}\right)-\omega$.

Investigating spectral stability of a relative equilibrium $\phi_{0}$ amounts to calculating the spectrum of $J \mathcal{L}$ where $\mathcal{L}$ is the Hessian of the energy. Since the energy is invariant under $G$, both $J \mathcal{L}$ and $\mathcal{L}$ will always have a nontrivial null space of dimension at least $\operatorname{dim} G \phi_{0}$. We denote by $X_{1}$ the range of $J \mathcal{L}$. The main result of $[7,8]$ states that the relative equilibrium $\phi_{0}$ will be nonlinearly orbitally stable if $\left.\mathcal{L}\right|_{X_{1}}$ is positive definite which implies that $\phi_{0}$ is a constrained minimizer.

Hence, let us consider the case where the reduced energy $\left.\mathcal{L}\right|_{X_{1}}$ has a finite number of negative eigenvalues. Note that $\phi_{0}$ is spectrally stable provided the spectrum of $J \mathcal{L}$ lies on the imaginary axis since eigenvalues of $J \mathcal{L}$ come in quadruples $\{ \pm \lambda, \pm \bar{\lambda}\}$. If $J \mathcal{L}$ has an eigenvalue $\lambda$ with positive real part, then the reduced energy restricted to the real invariant subspace $I_{\lambda}$ associated with the quadruplet $\{ \pm \lambda, \pm \bar{\lambda}\}$ is necessarily indefinite: indeed, if it were definite, its level sets would be spheres, and solutions in the subspace $I_{\lambda}$ to the linearized system

$$
v_{t}=J \mathcal{L} v
$$

which also conserves energy, are bounded, contradicting the assumption that $\lambda$ has positive real part. A useful term is therefore the Krein signature of an eigenvalue which is positive/negative or zero if the reduced energy restricted to the associated eigenspace is positive/negative definite or indefinite. Note that the above argument implies that, when purely imaginary eigenvalues collide on the imaginary axis, they can only leave the imaginary axis as a quadruplet when they have opposite Krein signature. This fact (and its converse, 
namely that colliding purely imaginary eigenvalues of opposite Krein signature will generically leave the imaginary axis) is more rigorously proved in $[3,6,13]$ (see $\S 2.2$ for details).

In light of the above discussion, we might expect that the number $n\left(\left.\mathcal{L}\right|_{X_{1}}\right)$ of negative eigenvalues of the reduced energy is a bound for the number $k_{\mathrm{u}}$ of unstable eigenvalues of $J \mathcal{L}$. In fact, we may expect that the difference between these two indices is the number $k_{\mathrm{i}}^{-}$of purely imaginary eigenvalues of $J \mathcal{L}$ with negative Krein signature. Our first result, Theorem 3.2, states that $k_{\mathrm{u}}+k_{\mathrm{i}}^{-} \leq \mathrm{n}\left(\left.\mathcal{L}\right|_{X_{1}}\right)$, while we prove in Theorem 3.3 that indeed

$$
k_{\mathrm{u}}+k_{\mathrm{i}}^{-}=\mathrm{n}\left(\left.\mathcal{L}\right|_{X_{1}}\right)
$$

provided $J$ is the canonical symplectic operator and $\mathcal{L}$ is diagonal so that

$$
J=\left(\begin{array}{cc}
0 & \mathbb{1} \\
-\mathbb{1} & 0
\end{array}\right), \quad \mathcal{L}=\left(\begin{array}{cc}
L_{+} & 0 \\
0 & L_{-}
\end{array}\right) .
$$

An expression for $\mathrm{n}\left(\left.\mathcal{L}\right|_{X_{1}}\right)$ in terms of $\mathrm{n}(\mathcal{L})$ is given in Theorem 3.1. We emphasize that the proofs of these results are based on the works [5-8] by Grillakis and coworkers; our contribution consists of an interpretation of their results in terms of the Krein signature. Our motivation for writing this paper was to give a short proof of (1.4) which generalizes several special cases that appeared recently in the literature (see below).

One consequence of the above results is that the number $k_{\mathrm{u}}+k_{\mathrm{i}}^{-}$can only change when the negative index of the energy changes. One common way for this to happen is when perturbations are added to the energy that break the symmetry $G$, a scenario that we refer to as forced symmetry breaking. Indeed, as outlined above, forced symmetry breaking reduces the dimension of the null space of $\mathcal{L}$, and of $J \mathcal{L}$, from $\operatorname{dim} G \phi_{0}$ to $\operatorname{dim} H \phi_{0}$, where $H$ is the subgroup of symmetries that are not broken by the perturbation. In Theorem 4.4, we calculate the perturbed eigenvalues of both $\mathcal{L}$ and $J \mathcal{L}$ near the origin, and collect various implications of this perturbation analysis for the count of $k_{\mathrm{u}}+k_{\mathrm{i}}^{-}$in $\S 5$. We then apply these results in $\S 6$ to forced symmetry breaking of weakly coupled nonlinear Schrödinger equations. It turns out that breaking the rotation symmetries induced by phase invariances will always lead to eigenvalues that are unstable or have negative Krein signature, while purely imaginary eigenvalues arising from breaking the translation symmetry will always have positive Krein signature.

Finally, we comment on related work. In simultaneous work, Pelinovsky and coworkers recently proved (1.4) for two specific PDEs under more restrictive hypotheses. Firstly, Pelinovsky established the count (1.4) in [17, Theorems 6-8] for the coupled NLS system (1.3) provided [17, Assumption 2.15] is met: this hypothesis requires that the linearization about the nonlinear wave has no eigenvalues that are embedded in the essential spectrum, that the algebraic multiplicity coincides with the geometric multiplicity for all non-zero eigenvalues and that the Evans function does not vanish at branch points. Theorems 3.1 and 3.3 and Remark 3.1 in our paper are applicable to (1.3) without these assumptions, while yielding the same conclusions. The authors of [2] proved (1.4) for linear NLS operators in dimension three or higher provided the branch points of the essential spectrum are not resonances. Again, this result, stated in [2, Theorem 2 and Corollary 2.7], follows directly from Theorem 3.3 in our paper without the hypothesis on the branch points. Forced symmetry breaking of a one-dimensional symmetry group in Hamiltonian systems has been studied previously by Skryabin [19]. Theorem 4.4 is a generalization to arbitrary symmetry groups. Kapitula [9] proved part of Theorem 4.4 using the Evans function but without computing the Krein signature.

Acknowledgments Kapitula would like to thank the Mathematical Research Institute at the Ohio State University for its support. Kapitula was partially supported by the NSF under grant DMS-0304982 and by the Army Research Office under grant ARO 45428-PH-HSI. Kevrekidis was partially supported by the NSF under grant DMS-0204585, by a Faculty Research Grant of the University of Massachusetts and by the Eppley Foundation for Research. Sandstede was partially supported by the NSF under grant DMS-0203854 and by an Alfred P Sloan Research Fellowship. 


\section{Formulation}

\subsection{General setup and hypotheses}

Let $U, V, X$ denote three real Hilbert spaces so that $U \subset X \subset V$ are densely and continuously embedded. Throughout this paper, we will only use the scalar product $\langle\cdot, \cdot\rangle$ on the space $V$. In particular, we have $U \subset X \subset V \subset X^{*}$ where $X^{*}$ denotes the dual space of $X$. Adjoint operators will always be taken with respect to the scalar product on $V$.

Let $G$ be a finite-dimensional abelian Lie group with Lie algebra $\mathfrak{g}$. We denote by $\exp (\omega)=\mathrm{e}^{\omega}$ for $\omega \in \mathfrak{g}$ the exponential map from $\mathfrak{g}$ into $G$. We assume that $T: G \rightarrow L(V)$ is a unitary representation of $G$ on $V$ so that $T^{\prime}(e)$ maps $\mathfrak{g}$ into the space of closed skew-symmetric operators on $V$ with domain $X$. We use the notation $T^{\prime}(e) \omega=: T_{\omega}$ for the generator of the semigroup $T\left(\mathrm{e}^{\omega t}\right)$. We assume that $U$ is contained in the domain of $T_{\omega}^{2}$. Note that $T_{\omega}$ is linear in $\omega \in \mathfrak{g}$. The group orbit $G u$ of an element $u \in X$ is defined by $G u:=\{T(g) u ; g \in G\}$. Lastly, we set $n=\operatorname{dim} G$.

We assume that $J: V \rightarrow V$ is a bounded, invertible and skew-symmetric linear operator so that $J^{*}=-J$. Furthermore, we assume that $E: X \rightarrow \mathbb{R}$ is a $\mathcal{C}^{2}$ functional such that the gradient $E^{\prime}(u)$ and the Hessian $E^{\prime \prime}(u)$, defined using the scalar product on $V$, are bounded as operators from $U$ into $\mathbb{R}$ and $V$, respectively, for each $u \in U$. We will frequently identify the Hessian $E^{\prime \prime}(u)$ on $U$ and the associated bilinear form on $X$.

Next, we introduce two compatibility assumptions. First, we assume that $E$ is invariant under $G$ so that

$$
E(T(g) u)=E(u)
$$

for all $u \in X$ and all $g \in G$. We also assume that

$$
T(g) J=J T\left(g^{-1}\right)^{*}
$$

for all $g \in G$. Lastly, for each $\omega \in \mathfrak{g}$, we define the bounded functional $Q_{\omega}: X \rightarrow \mathbb{R}$ by $Q_{\omega}(u):=$ $\frac{1}{2}\left\langle J^{-1} T_{\omega} u, u\right\rangle$. We see that the second derivative $Q_{\omega}^{\prime \prime}(u)=J^{-1} T_{\omega}$ is a bounded symmetric linear operator from $X$ to $V$. Note also that $Q_{\omega}$ is invariant ${ }^{1}$ under $G$ for each $\omega \in \mathfrak{g}$.

We are interested in the abstract Hamiltonian system

$$
\frac{\mathrm{d} u}{\mathrm{~d} t}=J E^{\prime}(u)
$$

Specifically, we are interested in relative equilibria of this system. These are solutions $u(t)$ whose time orbit is contained in the group orbit $G u_{0}$ so that $u(t) \in G u(0)$ for all $t$. Thus, $\phi \in X$ is a relative equilibrium if, and only if, there is a $\omega \in \mathfrak{g}$ so that $u(t)=T\left(\mathrm{e}^{\omega t}\right) \phi$ satisfies (2.1). In this context, relative equilibria are also called bound states or solitary waves. Substituting the ansatz $u(t)=T\left(\mathrm{e}^{\omega t}\right) \phi$ into $(2.1)$, we see that $\phi \in X$ is a relative equilibrium if, and only if,

$$
T_{\omega} \phi=J E^{\prime}(\phi),
$$

for some $\omega \in \mathfrak{g}$, that is, if and only if $\phi$ is a critical point of the functional $H_{\omega}:=E-Q_{\omega}$ for some $\omega \in \mathfrak{g}$. We assume that there exists a smooth family of bound states:

Hypothesis 2.1 (Relative equilibria) There is a non-empty open set $\Omega \subset \mathfrak{g}$ and a $\mathcal{C}^{1}$ function $\phi: \Omega \rightarrow U$, $\omega \mapsto \phi_{\omega}$ such that $\phi_{\omega}$ is a relative equilibrium, i.e., a critical point of $H_{\omega}=E-Q_{\omega}$ for each $\omega \in \Omega$. We assume that the isotropy subgroups $\left\{g \in G ; T(g) \phi_{\omega}=\phi_{\omega}\right\}$ are discrete for all $\omega$ so that the group orbits G $\phi_{\omega}$ have dimension $n$.

\footnotetext{
${ }^{1}$ This has been established in [8, Remark on p. 312] whose assumption is satisfied due to [18, Lemma 4.1]
} 
Since $G$ is abelian, the entire group orbit $T(g) \phi_{\omega}$ with $g \in G$ consists of relative equilibria with time evolution $T\left(\mathrm{e}^{\omega t}\right)$.

The Hessian of the energy at the relative equilibrium $\phi_{\omega}$ is defined by

$$
\mathcal{L}_{\omega}:=H_{\omega}^{\prime \prime}\left(\phi_{\omega}\right)=E^{\prime \prime}\left(\phi_{\omega}\right)-J^{-1} T_{\omega}
$$

which maps its domain $U$ into $V$. Note that the linearization of (2.1) about the relative equilibrium $\phi_{\omega}$ in the co-moving frame is given by $J \mathcal{L}_{\omega}$. As outlined in the introduction, we shall focus on the relation of the spectra of $\mathcal{L}_{\omega}$ and $J \mathcal{L}_{\omega}$. Due to the invariance of $H_{\omega}$ under the abelian group $G$, it follows from [8, p. 314] that the space

$$
Z=\operatorname{span}\left\{T_{\sigma} \phi_{\omega} ; \sigma \in \mathfrak{g}\right\}
$$

which is the tangent space of the group orbit $G \phi_{\omega}$ at $\phi_{\omega}$, is contained in the null space of $\mathcal{L}_{\omega}$. Hypothesis 2.1 implies that $\operatorname{dim} Z=\operatorname{dim} G=n$.

Hypothesis 2.2 (Spectral decomposition) We assume that the space $U$ is decomposed as a direct sum, $U=U_{\text {neg }} \oplus Z \oplus U_{\text {pos }}$, where $U_{\text {neg }}$ is a finite-dimensional subspace such that $\left\langle u, \mathcal{L}_{\omega} u\right\rangle<0$ for all $u \in U_{\text {neg }}$ with $u \neq 0$, and $U_{\text {pos }}$ is a closed subspace so that there is a $\delta>0$ such that $\left\langle u, \mathcal{L}_{\omega} u\right\rangle \geq \delta|u|_{X}^{2}$ for all $u \in U_{\text {pos }}$.

For any bounded, self-adjoint linear operator $A$ from $U$ to $V$, we denote by $z(A)$ the dimension of its null space and by $\mathrm{n}(A)$ and $\mathrm{p}(A)$ the dimensions of the maximal subspaces of $U$ on which $A$ is negative and positive definite, respectively. In particular, if $\mathrm{n}(A)$ is finite, then it coincides with the number of negative eigenvalues of $A$. Hence, as outlined in [8, p. 314], the null space of $\mathcal{L}_{\omega}$ is equal to $Z$, and the number of negative eigenvalues of $\mathcal{L}_{\omega}$ is equal to $\operatorname{dim} U_{\text {neg. }}$.

We shall often consider the following, more special situation that arises frequently in applications.

Hypothesis 2.3 (Canonical symplectic structure) We assume that each of the Hilbert spaces $U, X, V$ is the direct product of two closed isomorphic subspaces. Writing operators as $2 \times 2$ blocks that respect this decomposition, we assume that

$$
J=\left(\begin{array}{rr}
0 & \mathbb{1} \\
-\mathbb{1} & 0
\end{array}\right), \quad \mathcal{L}_{\omega}=\left(\begin{array}{cc}
L_{+} & 0 \\
0 & L_{-}
\end{array}\right)
$$

and that $Z=Z_{+} \times Z_{-}$is a direct product. Furthermore, we assume that there is a self-adjoint, strictly positive operator $\breve{L}$ so that both $L_{+}$and $L_{-}$are relatively compact perturbations of $\check{L}$.

Throughout this paper, we shall always assume that Hypotheses 2.1 and 2.2 are met. Whenever we make use of Hypothesis 2.3, we will indicate so.

\subsection{The Krein signature}

As already mentioned, we are interested in the relation of the spectra of $\mathcal{L}_{\omega}$ and $J \mathcal{L}_{\omega}$. Specifically, we wish to establish a connection between the number $\mathrm{n}\left(\mathcal{L}_{\omega}\right)$ of negative eigenvalues of the energy $\mathcal{L}_{\omega}$ and the number of eigenvalues of the linearization $J \mathcal{L}_{\omega}$ that have negative Krein signature. Roughly speaking, the Krein signature of an eigenvalue $\lambda$ of $J \mathcal{L}_{\omega}$ measures whether the energy $\mathcal{L}_{\omega}$ is positive definite, negative definite or indefinite when restricted to the generalized real eigenspace associated with the eigenvalues $\pm \lambda$ and $\pm \bar{\lambda}$.

To be precise, assume that $\lambda \in \mathbb{C}$ is an eigenvalue of $J \mathcal{L}_{\omega}$. Since $J$ is skew-symmetric and $\mathcal{L}_{\omega}$ is symmetric, it follows that $\pm \lambda$ and $\pm \bar{\lambda}$ are also eigenvalues of $J \mathcal{L}_{\omega}$. Let $I_{\lambda}$ denote the real invariant subspace of $U$ that is the sum of the generalized eigenspaces associated with the eigenvalues $\pm \lambda$ and $\pm \bar{\lambda}$. Following [13, p. 144-145], 
we say that $\lambda$ has positive or negative Krein signature if $\mathcal{L}_{\omega}$ restricted to $I_{\lambda}$ is positive or negative definite and that it has zero Krein signature if $\mathcal{L}_{\omega}$ restricted to $I_{\lambda}$ is indefinite.

It is a consequence of [13, Lemma 5(ii)] that the Krein signature of $\lambda$ is zero if $\lambda$ has non-zero real part. The Krein signature is also zero if the geometric and algebraic multiplicity of $\lambda$ as an eigenvalue of $J \mathcal{L}_{\omega}$ are not equal, i.e., if $\lambda$ has a non-trivial Jordan block.

If $\lambda \in \mathrm{i} \mathbb{R}$ is a simple, non-zero, purely imaginary eigenvalue of $J \mathcal{L}_{\omega}$ with complex eigenfunction $v=v_{1}+\mathrm{i} v_{2}$, then we have $I_{\lambda}=\operatorname{span}\left\{v_{1}, v_{2}\right\}$. In this case, the Krein signature of $\lambda$ is non-zero and is, in fact, given by $\operatorname{sign}\left\langle v, \mathcal{L}_{\omega} v\right\rangle=\operatorname{sign}\left\langle v_{1}, \mathcal{L}_{\omega} v_{1}\right\rangle$ (see [13, Proof of Lemma 5(i)]). More generally, the results stated in [13, Lemmas 4 and 5(i)] imply that the energy $\mathcal{L}_{\omega}$, considered as a bilinear form on $I_{\lambda}$, is non-degenerate and has an even number of positive and negative eigenvalues whenever $\lambda \in i \mathbb{R}$ is a non-zero purely imaginary eigenvalue of $J \mathcal{L}_{\omega}$. In this case, we denote by $2 \mathrm{n}_{\lambda}^{-}$the number of negative squares of $\mathcal{L}_{\omega}$ restricted to $I_{\lambda}$.

Definition 2.4 Consider the eigenvalues of $J \mathcal{L}_{\omega}$ on $X$. Counting multiplicity, we denote by $k_{\mathrm{r}}$ the number of positive real eigenvalues of $J \mathcal{L}_{\omega}$ and by $k_{\mathrm{c}}$ the number of quadruplets $\{ \pm \lambda, \pm \bar{\lambda}\}$ of eigenvalues $\lambda$ of $J \mathcal{L}_{\omega}$ that are neither in $\mathbb{R}$ nor in $\mathrm{i} \mathbb{R}$. Lastly, we denote by $k_{\mathrm{i}}^{-}$the sum of the numbers $\mathrm{n}_{\lambda}^{-}$where $\lambda$ runs through all purely imaginary eigenvalues with positive imaginary part.

The relevance of the Krein signature is that non-zero eigenvalues of $J \mathcal{L}_{\omega}$ on the imaginary axis can leave the imaginary axis only when eigenvalues with opposite Krein signature collide (see [13, Theorem on p. 146] for systems without symmetries and [3, §4] for equivariant equations) or if an eigenvalue with negative sign is originally embedded in the continuous spectrum (see [6, Theorem 2.4]). We refer to [13] for more detailed background information about the material reviewed in this section.

\section{Counting eigenvalues with negative Krein signature}

Throughout this section, we assume that Hypotheses 2.1 and 2.2 are satisfied.

\subsection{The reduced Hamiltonian}

Recall that the null space of the operator $\mathcal{L}_{\omega}$ is given by $Z=\operatorname{span}\left\{T_{\sigma} \phi_{\omega} ; \sigma \in \mathfrak{g}\right\}$ which, by assumption, has dimension $n=\operatorname{dim} G$. We fix a basis $\left\{\sigma_{1}, \ldots, \sigma_{n}\right\}$ of $\mathfrak{g}$, and denote by $D \in \mathbb{R}^{n \times n}$ the symmetric matrix with entries

$$
D_{i j}=\left\langle\partial_{\sigma_{i}} \phi_{\omega}, \mathcal{L}_{\omega} \partial_{\sigma_{j}} \phi_{\omega}\right\rangle .
$$

Since $J$ is invertible by assumption, we see that $\lambda=0$ is an eigenvalue of $J \mathcal{L}_{\omega}$ with geometric multiplicity $n$. We now have

$$
J \mathcal{L}_{\omega} T_{\sigma_{j}} \phi_{\omega}=0, \quad J \mathcal{L}_{\omega} \partial_{\sigma_{j}} \phi_{\omega}=T_{\sigma_{j}} \phi_{\omega}, \quad \forall j
$$

where the left equation follows from [8, p. 314] upon using that $G$ is abelian, while the right equation follows by differentiating (2.2). Thus, the algebraic multiplicity of $\lambda=0$ as an eigenvalue of $J \mathcal{L}_{\omega}$ is at least $2 n$. Using that the null space of the adjoint of $J \mathcal{L}_{\omega}$ is spanned by

$$
J^{-1} T_{\sigma_{j}} \phi_{\omega}=\mathcal{L}_{\omega} \partial_{\sigma_{j}} \phi_{\omega}, \quad \forall j,
$$

it is not hard to prove that the algebraic multiplicity of $\lambda=0$ is equal to $2 n$ if, and only if, the matrix $D$ is invertible. We denote by $X_{1}$,

$$
X_{1}=\left\{u \in X ;\left\langle u, \mathcal{L}_{\omega} \partial_{\sigma_{i}} \phi_{\omega}\right\rangle=0\right\},
$$

the range of $J \mathcal{L}_{\omega}$. 
Theorem 3.1 ([8, Theorem 3.1]) Assume that Hypotheses 2.1 and 2.2 are met. The operator $\mathcal{L}_{\omega}$ restricted to $X_{1}$ has

$$
\mathrm{n}\left(\left.\mathcal{L}_{\omega}\right|_{X_{1}}\right)=\mathrm{n}\left(\mathcal{L}_{\omega}\right)-\mathrm{n}(D)-\mathrm{z}(D)
$$

negative eigenvalues, and its null space has dimension

$$
\mathrm{z}\left(\left.\mathcal{L}_{\omega}\right|_{X_{1}}\right)=\mathrm{z}(D)+\operatorname{dim} G .
$$

Finally, if $\mathrm{n}\left(\mathcal{L}_{\omega} \mid X_{1}\right)=0$ and $\mathrm{z}(D)=0$, then $\phi_{\omega}$ is a local minimizer of $H_{\omega}$.

Proof. [8, Theorem 3.1] gives our theorem upon using that, in the notation of [8], we have $\mathrm{z}\left(\mathcal{L}_{\omega}\right)=\operatorname{dim} G$, $d^{\prime \prime}=-D$, and $z_{0}:=\operatorname{dim}\left\{\sigma \in \mathfrak{g} ; T_{\sigma} \phi_{\omega}=0\right\}=0$ is equal to the dimension of the isotropy subgroup of $\phi_{\omega}$ which we assumed to be zero.

\subsection{General setup}

Theorem 3.1 gives the number of unstable eigenvalues of the reduced energy $\left.\mathcal{L}_{\omega}\right|_{X_{1}}$. In this section, we show how this result can be used to estimate the number of eigenvalues of the linearization $J \mathcal{L}_{\omega}$ about the wave $\phi_{\omega}$ that have either negative Krein signature or are unstable.

Theorem 3.2 If Hypotheses 2.1 and 2.2 are met, then

$$
k_{\mathrm{r}}+2 k_{\mathrm{c}}+2 k_{\mathrm{i}}^{-} \leq \mathrm{n}\left(\mathcal{L}_{\omega}\right)-\mathrm{n}(D)-\mathrm{z}(D),
$$

where the left-hand side has been introduced in Definition 2.4. In particular, the left-hand side is finite.

Note that the preceding theorem is sharper than [8, Theorem 5.8]. We remark that $[8$, Theorem 5.1] asserts that $k_{\mathrm{r}} \geq 1$ whenever $\mathrm{z}(D)=0$ and $\mathrm{n}\left(\mathcal{L}_{\omega}\right)-\mathrm{n}(D)$ is odd.

Proof. Let $\left\{\lambda_{j} ; j=1, \ldots, k\right\}$ denote the eigenvalues of $J \mathcal{L}_{\omega}$ that are either positive and real, or nonzero and purely imaginary with negative Krein signature, or lie neither in $\mathbb{R}$ nor in $i \mathbb{R}$. For each of these eigenvalues, we obtain the real invariant subspace $I_{\lambda_{j}}$ that we defined in $\S 2.2$. Consider the space $I_{\lambda}$ for an eigenvalue $\lambda$ with non-zero real part. In this case, [13, Lemma 5(ii)] implies that there is a subspace $I_{\lambda}^{-}$of dimension $\left(\operatorname{dim} I_{\lambda}\right) / 2$ so that $\mathcal{L}_{\omega}$ restricted to $I_{\lambda}^{-}$is negative definite. If, on the other hand, $\lambda$ is a non-zero purely imaginary eigenvalue with negative Krein signature, then $\mathcal{L}_{\omega}$ restricted to $I_{\lambda}^{-}:=I_{\lambda}$ is negative definite.

We now denote by $I^{-}$the direct sum of the subspaces $I_{\lambda_{j}}^{-}$that we obtained in the preceding discussion. It is a consequence of [13, Lemma 3] that $\mathcal{L}_{\omega}$ restricted to $I^{-}$is also negative definite. Furthermore, since $\lambda_{j} \neq 0$ for all $j$, we have $I_{\lambda_{j}} \subset X_{1}$ for all $j$ and therefore also $I^{-} \subset X_{1}$. This implies that $\operatorname{dim} I^{-} \leq \mathrm{n}\left(\left.\mathcal{L}_{\omega}\right|_{X_{1}}\right)$. Counting the dimension of $I^{-}$and using Theorem 3.1 to get an expression for $\mathrm{n}\left(\left.\mathcal{L}_{\omega}\right|_{X_{1}}\right)$ completes the proof.

\subsection{Canonical symplectic structure}

Theorem 3.2 is not entirely satisfactory as it gives only an upper bound for $k_{\mathrm{r}}+2 k_{\mathrm{c}}+2 k_{\mathrm{i}}^{-}$instead of an exact count. In this section, we establish such an exact count provided Hypothesis 2.3 is satisfied. Thus, throughout this section, we shall assume that Hypotheses 2.1, 2.2 and 2.3 are met.

We then have $X=\check{X} \oplus \check{X}$,

$$
J=\left(\begin{array}{rr}
0 & \mathbb{1} \\
-\mathbb{1} & 0
\end{array}\right), \quad \mathcal{L}_{\omega}=\left(\begin{array}{cc}
L_{+} & 0 \\
0 & L_{-}
\end{array}\right), \quad J \mathcal{L}_{\omega}=\left(\begin{array}{cc}
0 & L_{-} \\
-L_{+} & 0
\end{array}\right)
$$


and $Z=Z_{+} \times Z_{-} \subset \check{X} \times \check{X}$ with $\operatorname{dim} Z_{ \pm}=z_{ \pm}$where $z_{+}+z_{-}=n$. After adjusting the basis $\left\{\sigma_{i}\right\}$ of $\mathfrak{g}$ and using (3.2) and (3.5), we see that there are elements $\phi_{i}^{+}, \phi_{j}^{-}, \psi_{i}^{+}, \psi_{j}^{-} \in \check{X}, \check{\phi}_{i}^{+} \in Z_{+}$and $\check{\phi}_{j}^{-} \in Z_{-}$, defined for $i=1, \ldots, z_{+}$and $j=1, \ldots, z_{-}$, such that

$$
T_{\sigma_{i}} \phi_{\omega}=\left(\begin{array}{c}
\phi_{i}^{+} \\
0
\end{array}\right), \quad T_{\sigma_{z_{+}+j}} \phi_{\omega}=\left(\begin{array}{c}
0 \\
\phi_{j}^{-}
\end{array}\right), \quad \partial_{\sigma_{i}} \phi_{\omega}=\left(\begin{array}{c}
\check{\phi}_{i}^{+} \\
\psi_{i}^{+}
\end{array}\right), \quad \partial_{\sigma_{z_{+}+j}} \phi_{\omega}=\left(\begin{array}{c}
\psi_{j}^{-} \\
\check{\phi}_{j}^{-}
\end{array}\right)
$$

where $i=1, \ldots, z_{+}$and $j=1, \ldots, z_{-}$. It is then straightforward to check that the matrix $D$, defined in (3.1)

$$
D_{i j}=\left\langle\partial_{\sigma_{i}} \phi_{\omega}, \mathcal{L}_{\omega} \partial_{\sigma_{j}} \phi_{\omega}\right\rangle
$$

is of the form

$$
D=\left(\begin{array}{cc}
D_{-} & 0 \\
0 & D_{+}
\end{array}\right), \quad D_{-} \in \mathbb{R}^{z_{+} \times z_{+}}, \quad D_{+} \in \mathbb{R}^{z_{-} \times z_{-}},
$$

where the entries of $D_{+}$and $D_{-}$are given by

$$
\left[D_{-}\right]_{i j}=\left\langle\psi_{i}^{+}, L_{-} \psi_{j}^{+}\right\rangle=\left\langle\psi_{i}^{+}, \phi_{j}^{+}\right\rangle, \quad\left[D_{+}\right]_{i j}=\left\langle\psi_{i}^{-}, L_{+} \psi_{j}^{-}\right\rangle=\left\langle\psi_{i}^{-}, \phi_{j}^{-}\right\rangle .
$$

Theorem 3.3 Assume that Hypotheses 2.1, 2.2 and 2.3 are satisfied and that the matrix D is invertible.

We then have

$$
k_{\mathrm{r}}+2 k_{\mathrm{c}}+2 k_{\mathrm{i}}^{-}=\mathrm{n}\left(\mathcal{L}_{\omega}\right)-\mathrm{n}(D)=\mathrm{n}\left(L_{+}\right)+\mathrm{n}\left(L_{-}\right)-\mathrm{n}\left(D_{+}\right)-\mathrm{n}\left(D_{-}\right),
$$

where the left-hand side has been introduced in Definition 2.4.

Thus, if all non-zero eigenvalues are simple, Theorem 3.3 states that the number of unstable eigenvalues in the open right half-plane plus the number of non-zero, purely imaginary eigenvalues with negative Krein signature is equal to the number of negative directions of the reduced energy. The following remark gives a lower bound on the number of unstable eigenvalues using [5].

Remark 3.1 Under the hypotheses of Theorem 3.3, we have the lower bound

$$
k_{\mathrm{r}} \geq\left|\mathrm{n}\left(L_{+}\right)-\mathrm{n}\left(L_{-}\right)-\mathrm{n}\left(D_{+}\right)+\mathrm{n}\left(D_{-}\right)\right|
$$

for the number of unstable real eigenvalues. Furthermore, we have

$$
k_{\mathrm{r}}=\left|\mathrm{n}\left(L_{+}\right)-\mathrm{n}\left(D_{+}\right)\right|, \quad k_{\mathrm{c}}=k_{\mathrm{i}}^{-}=0, \quad \text { provided } \quad \mathrm{n}\left(L_{-}\right)=\mathrm{n}\left(D_{-}\right) .
$$

The bound (3.9) follows immediately from [5, Theorem 2.1] and Lemma 3.2 below, while (3.10) follows from [5, Corollary 1.1] together with Lemma 3.2. [We remark that [5, Corollary 1.1] is true only if, in the notation of [5], $R$ and $S$ are both invertible; we prove this for our operators in Lemma 3.2 below].

\subsection{Proof of Theorem 3.3}

We prove Theorem 3.3 and Remark 3.1 in the following, slightly more general setting: We do not assume equivariance with respect to any symmetry but require only that the null spaces $Z_{ \pm}$of $L_{ \pm}$satisfy $Z_{+} \subset$ $\operatorname{Rg}\left(L_{-}\right)$and $Z_{-} \subset \operatorname{Rg}\left(L_{+}\right)$; note that equation (3.6) shows that this is true in the equivariant context. We can therefore find finite-dimensional spaces $Y_{ \pm} \in \hat{X}$ with $\operatorname{dim} Y_{ \pm}=z_{ \pm}$so that $Z_{+}=L_{-}\left(Y_{+} \oplus Z_{-}\right)$and $Z_{-}=L_{+}\left(Y_{-} \oplus Z_{+}\right)$. Next, choose bases $\left\{y_{1}^{ \pm}, \ldots, y_{z_{ \pm}}^{ \pm}\right\}$of $Y_{ \pm}$and define the matrices $D_{ \pm}$via

$$
\left[D_{+}\right]_{i j}=\left\langle y_{i}^{-}, L_{+} y_{j}^{-}\right\rangle, \quad\left[D_{-}\right]_{i j}=\left\langle y_{i}^{+}, L_{-} y_{j}^{+}\right\rangle .
$$

Note that this definition of $D$ coincides with the one given in (3.7) in the situation considered in $\S 3.3$.

We set $\check{Z}=Z_{+}+Z_{-}$and denote by $P$ and $P_{ \pm}$the orthogonal projections in $\check{X}$ onto $\check{Z}^{\perp}$ and $\check{Z}_{ \pm}^{\perp}$, respectively. 
Lemma 3.2 Assume that Hypotheses 2.1, 2.2 and 2.3 are satisfied. We then have $Z_{+} \perp Z_{-}$which implies $\check{Z}=Z_{+} \oplus Z_{-}$and $P=P_{+} P_{-}=P_{-} P_{+}$. Furthermore, if the matrix $D$ is invertible, then the operators $P L_{+} P$ and $P L_{-} P$ are invertible on $\check{Z}^{\perp}$ and

$$
\mathrm{n}\left(P L_{+} P\right)=\mathrm{n}\left(L_{+}\right)-\mathrm{n}\left(D_{-}\right), \quad \mathrm{n}\left(P L_{-} P\right)=\mathrm{n}\left(L_{-}\right)-\mathrm{n}\left(D_{+}\right) .
$$

Proof. By definition, the vectors $\phi_{i}^{ \pm}=L_{\mp} y_{i}^{ \pm}$form a basis of $Z_{ \pm}$. The identity

$$
\left\langle\phi_{i}^{+}, \phi_{j}^{-}\right\rangle=\left\langle L_{-} y_{i}^{+}, \phi_{j}^{-}\right\rangle=\left\langle y_{i}^{+}, L_{-} \phi_{j}^{-}\right\rangle=0
$$

proves that $Z_{+} \perp Z_{-}$. Next, we remark that $L_{+}$and $L_{-}$are Fredholm operators with index zero by Hypothesis 2.2. Thus, $P L_{+} P$ and $P L_{-} P$ are invertible if, and only if, their null spaces are trivial. We prove that $P L_{+} P$ has trivial null space on $\check{Z}^{\perp}$ if, and only if, $D_{-}$is invertible. Thus, assume that $P L_{+} P u=0$ for some $u \in \check{Z}^{\perp}$, which means that $L_{+} u \in Z_{-}$. The preimage $L_{+}^{-1} Z_{-}$of $Z_{-}$under $L_{+}$is given by $Y_{-}+Z_{+}$ where $Y_{-}=\operatorname{span}\left\{\psi_{j}^{-} ; j=1, \ldots, z_{-}\right\}$. Thus, the null space of $P L_{+} P$ is trivial if, and only if, $\left(Y_{-}+Z_{+}\right) \cap \check{Z}^{\perp}$ is trivial. On the other hand, if the matrix $D_{-}$is invertible, then (3.7) implies that $Y_{-} \cap Z_{-}^{\perp}$ is trivial, which in turn shows that $\left(Y_{-}+Z_{+}\right) \cap \check{Z}^{\perp}$ is trivial. This proves the statement concerning the invertibility of $P L_{+} P$ and $P L_{-} P$. Lastly, (3.5) implies that the range $X_{1}$ of $J \mathcal{L}_{\omega}$ is given by $X_{1}=\operatorname{Rg}\left(L_{-}\right) \times \operatorname{Rg}\left(L_{+}\right)$. Furthermore, we have

$$
\left.\mathcal{L}_{\omega}\right|_{X_{1}}=\left(\begin{array}{cc}
L_{+} \mid \operatorname{Rg}\left(L_{-}\right) & 0 \\
0 & \left.L_{-}\right|_{\operatorname{Rg}\left(L_{+}\right)}
\end{array}\right)=\left(\begin{array}{cc}
\left.L_{+}\right|_{Z_{-}^{\perp}} & 0 \\
0 & \left.L_{-}\right|_{Z_{+}^{\perp}}
\end{array}\right) .
$$

Using these expressions together with [15, Lemma 6 and Theorem 2] for each of the operators on the diagonal proves the index formulas (3.11).

From now on, we assume that $D$ is invertible. Thus, $P L_{+} P$ and $P L_{-} P$ are both invertible as operators from $\check{Z}^{\perp}$ into itself. We define $R:=P L_{+} P$ and $S:=\left(P L_{-} P\right)^{-1}$ both considered on $\check{Z}^{\perp}$. Of interest is then the generalized eigenvalue problem

$$
[R-z S] u=0
$$

for $u \in \check{Z}^{\perp}$ and $z \in \mathbb{C}$.

Lemma 3.3 Assume that Hypotheses 2.1, 2.2 and 2.3 are satisfied and that the matrix $D$ is invertible. In this situation, non-zero eigenvalues $z$ of (3.12) on $\check{Z}^{\perp}$ and non-zero eigenvalues $\lambda$ of $J \mathcal{L}_{\omega}$ on $X$ are in 1:1 correspondence (including their geometric and algebraic multiplicity) via $z=-\lambda^{2}$.

Proof. We prove that non-zero eigenvalues of (3.12) on $\check{Z}^{\perp}$ and $J \mathcal{L}_{\omega}$ on $X$ are in 1:1 correspondence. Thus, suppose that $(u, v) \in \check{X} \times \check{X}$ satisfies

$$
L_{-} v=\lambda u, \quad-L_{+} u=\lambda v
$$

for some $\lambda \neq 0$. We conclude that $u \in Z_{-}^{\perp}$ and $v \in Z_{+}^{\perp}$ so that (3.13) and

$$
P_{-} L_{-} P_{-} v=\lambda P_{-} u, \quad-P_{+} L_{+} P_{+} u=\lambda P_{+} v
$$

with $u \in Z_{-}^{\perp}$ and $v \in Z_{+}^{\perp}$ are equivalent. Due to these restrictions on $u$ and $v,(3.13)$ is in fact equivalent to

$$
P_{-} L_{-} P v=\lambda P_{-} u, \quad-P_{+} L_{+} P u=\lambda P_{+} v
$$

with $u \in Z_{\perp}^{\perp}$ and $v \in Z_{+}^{\perp}$. Lastly, upon writing $u=\check{u}+\phi^{+}$and $v=\check{v}+\phi^{-}$with $\check{u}, \check{v} \in \check{Z}^{\perp}$ and $\phi^{ \pm} \in Z_{ \pm}$, and projecting the two equations in (3.15) using $P_{+}$and $P_{-}$, we see that (3.13) is equivalent to

$$
P L_{-} P \check{v}=\lambda \check{u}, \quad-P L_{+} P \check{u}=\lambda \check{v}
$$


and

$$
\left(1-P_{+}\right) P_{-} L_{-} P \check{v}=\lambda \phi^{+}, \quad-\left(1-P_{-}\right) P_{+} L_{+} P \check{u}=\lambda \phi^{-} .
$$

Note that we used $Z_{+} \perp Z_{-}$. Using the definition of $R$ and $S$, we see that (3.16) is equivalent to $\left[R+\lambda^{2} S\right] \check{u}=0$ which is exactly (3.12), while (3.17) uniquely determines $\phi^{+}$and $\phi^{-}$. The claim regarding the algebraic multiplicity can be proved in the same fashion.

Suppose now that $(p, q) \in \check{X} \times \check{X}$ is an eigenfunction associated with the non-zero purely imaginary eigenvalue $\lambda=\mathrm{i} \mu$ of $J \mathcal{L}_{\omega}$ so that

$$
J \mathcal{L}_{\omega}\left(\begin{array}{c}
p \\
q
\end{array}\right)=\left(\begin{array}{cc}
0 & L_{-} \\
-L_{+} & 0
\end{array}\right)\left(\begin{array}{l}
p \\
q
\end{array}\right)=\mathrm{i} \mu\left(\begin{array}{l}
p \\
q
\end{array}\right)
$$

and $\left[R-\mu^{2} S\right] p=0$. We may assume that $p$ is real and $q$ is purely imaginary. A straightforward calculation shows that the signature of $\mathcal{L}_{\omega}$ restricted to the real invariant eigenspace associated with $(p, q)$ is then given by

$$
\left\langle\mathcal{L}_{\omega}\left(\begin{array}{c}
p \\
q
\end{array}\right),\left(\begin{array}{c}
p \\
q
\end{array}\right)\right\rangle=2 \mu^{2}\langle S p, p\rangle=2 \mu^{2}\left\langle R^{-1} q, q\right\rangle .
$$

Thus, we shall concentrate on the generalized eigenvalue problem

$$
\begin{array}{r}
{[R-z S] p=0} \\
\langle S p, p\rangle \leq 0 .
\end{array}
$$

In [6, Theorem 2.3] it is stated that (3.19)-(3.20) has precisely $\mathrm{n}(S)$ pairs of solutions $\left(z_{i}, p_{i}\right)$ and $\left(\bar{z}_{i}, \bar{p}_{i}\right)$. Furthermore, if $z \in \mathbb{R}$ corresponds to a Jordan block, or if $\operatorname{Im} z \neq 0$, then $\langle S p, \bar{p}\rangle=0$. Alternatively, we can consider

$$
\begin{aligned}
{\left[S^{-1}-z R^{-1}\right] q } & =0 \\
\left\langle R^{-1} q, q\right\rangle & \leq 0 .
\end{aligned}
$$

Applying the same theorem, we see that (3.21)-(3.22) has precisely $\mathrm{n}(R)=\mathrm{n}\left(R^{-1}\right)$ pairs of solutions $\left(z_{j}, q_{j}\right)$ and $\left(\bar{z}_{j}, \bar{q}_{j}\right)$.

Note that $(z, p)$ satisfies $[R-z S] p=0$ if, and only if, $(z, q)$ satisfies $\left[S^{-1}-z R^{-1}\right] q=0$ where $q=S p$. In this situation, we therefore have

$$
\langle S p, p\rangle=\left\langle q, S^{-1} q\right\rangle=\left\langle S^{-1} q, q\right\rangle=z\left\langle R^{-1} q, q\right\rangle .
$$

Since the scalar products are real, we have shown that

$$
\langle S p, p\rangle=\left\langle R^{-1} q, q\right\rangle=0
$$

or $z \in \mathbb{R}$ (or both). In summary, solutions $(z, p)$ and $(z, q)$ of (3.19) and (3.21) are in 1:1 correspondence via $q=S p$, and we have (3.23) whenever $z \notin \mathbb{R}$.

We now account for all the possibilities of $(z, p)$ and $(z, q)$ not only solving (3.19) and (3.21) but also (3.20) and (3.22). As in [6], we will denote the set of equations (3.19)-(3.20) by (S) and equations (3.21)-(3.22) by (R). Also, $p$ and $q$ are always related via $q=S p$.

- If $z>0$, then $(z, p, q)$ satisfies (S) and (R), and we have $\langle S p, p\rangle=\left\langle R^{-1} q, q\right\rangle \leq 0$. In particular, the eigenvalues have zero or negative signature.

- If $\operatorname{Im} z \neq 0$, or $z \in \mathbb{R}$ corresponds to a Jordan block, then $(z, p, q)$ satisfies (S) and (R), and we have $\langle S p, p\rangle=\left\langle R^{-1} q, q\right\rangle=0$ (see above). 
- If $z<0$, then we have the following exclusive alternative: Either $\langle S p, p\rangle=\left\langle R^{-1} q, q\right\rangle=0$, in which case $(z, p, q)$ satisfies $(\mathrm{S})$ and $(\mathrm{R})$, or else $\operatorname{sign}\langle S p, p\rangle=-\operatorname{sign}\left\langle R^{-1} q, q\right\rangle \neq 0$ in which case $(z, p, q)$ solves either $(\mathrm{S})$ or $(\mathrm{R})$ but not both.

We count the solutions in each category. To summarize, if

- $z>0$ with negative signature $\left(\ell_{1}\right.$ solutions $)$,

- $\operatorname{Im} z \neq 0\left(\ell_{2}\right.$ solutions $)$

- $z \in \mathbb{R}$ corresponds to a Jordan block ( $\ell_{3}$ solutions), or

- $z<0$ with vanishing signature $\left(\ell_{4}\right.$ solutions $)$,

then $(z, p, q)$ satisfies both $(\mathrm{S})$ and $(\mathrm{R})$. On the other hand, if $z<0$ has non-zero signature, then $(z, p, q)$ satisfies either ( $\mathrm{S}$ ) or (R) but not both (so that we have $\ell_{5}$ solutions with $\ell_{5}=\ell_{5}^{R}+\ell_{5}^{S}$ with the obvious notation). Recall that we have precisely $\mathrm{n}(R)$ solutions to $(\mathrm{R})$ and precisely $\mathrm{n}(S)$ solutions to (S). Hence, we have

$$
\mathrm{n}(R)=\ell_{1}+\ell_{2}+\ell_{3}+\ell_{4}+\ell_{5}^{R}, \quad \mathrm{n}(S)=\ell_{1}+\ell_{2}+\ell_{3}+\ell_{4}+\ell_{5}^{S},
$$

which gives the count

$$
\mathrm{n}(R)+\mathrm{n}(S)=2 \ell_{1}+2 \ell_{2}+2 \ell_{3}+2 \ell_{4}+\ell_{5} .
$$

Lastly, Lemma 3.3 shows that the right-hand side counts, with multiplicity, $k_{\mathrm{r}}+2 k_{\mathrm{c}}+2 k_{\mathrm{i}}^{-}$. Using Lemma 3.2 for the left-hand side of (3.24) then completes the proof of Theorem 3.3.

\section{Forced symmetry breaking: perturbation analysis}

The results presented in the previous sections provide counts of unstable eigenvalues and purely imaginary eigenvalues with negative Krein signature of the linearization $J \mathcal{L}_{\omega}$ about relative equilibria in Hamiltonian nonlinear PDEs that are equivariant under the abelian Lie group $G$. In addition to these eigenvalues, there are always $2 n$ eigenvalues at $\lambda=0$ that are enforced by the symmetry group $G$ where $n=\operatorname{dim} G$. If the symmetry $G$ is broken, some of the eigenvalues at $\lambda=0$ may move away from the origin and possibly generate additional unstable eigenvalues or eigenvalues with negative Krein signature. In this section, we investigate this scenario.

Hypothesis 4.1 Suppose that $H \leq G$ is a subgroup of $G$ with Lie algebra $\mathfrak{h}$ and dimension $\operatorname{dim} H=h$. We assume that the energy is of the form $E=E_{0}+\epsilon E_{1}$ for $0<\epsilon \ll 1$ where $E_{0}$ is invariant under $G$ and $E_{1}$ is invariant under H. Furthermore, we assume that Hypotheses 2.1 and 2.2 are met for $\epsilon=0$.

We denote by $D_{G}$ the matrix defined in (3.1) and choose bases $\left\{\tau_{1}, \ldots, \tau_{h}\right\}$ of $\mathfrak{h}$ and $\left\{\tau_{1}^{\perp}, \ldots, \tau_{n-h}^{\perp}\right\}$ of a complement $\mathfrak{h}^{\perp}$ of $\mathfrak{h}$ in $\mathfrak{g}$.

\subsection{Persistence of relative equilibria}

First, we consider the persistence of relative equilibria for $\epsilon>0$. Pick a relative equilibrium $\phi_{\omega}^{0}$ for $\epsilon=0$ with $\omega \in \mathfrak{h}$ and define

$$
E_{\text {red }}: \quad \mathfrak{h}^{\perp} \longrightarrow \mathbb{R}, \quad \tau \longmapsto E_{1}\left(T(\exp (\tau)) \phi_{\omega}^{0}\right)
$$

which evaluates the perturbation $E_{1}$ along the directions in $G \phi_{\omega}^{0}$ that are transverse to $H \phi_{\omega}^{0}$. We can now formulate the following persistence result that can be proved easily using equivariant Lyapunov-Schmidt reduction. 
Proposition 4.2 Assume that Hypothesis 4.1 is satisfied and $D_{G}$ is invertible. Let $\phi_{\omega}^{0}$ be a relative equilibrium defined for $\epsilon=0$ with $\omega \in \mathfrak{h}$. If $\tau=0$ is a nondegenerate critical point of the associated reduced energy $E_{\mathrm{red}}$, then there exists a smooth family $\phi_{\omega}(\epsilon)$ of relative equilibria for $0<\epsilon \ll 1$ with $\phi_{\omega}(0)=\phi_{\omega}^{0}$.

Note that, by definition, $\tau=0$ is a nondegenerate critical point of $E_{\text {red }}$ if

$$
\left\langle T_{\tau_{j}^{\perp}} \phi_{\omega}^{0}, E_{1}^{\prime}\left(\phi_{\omega}^{0}\right)\right\rangle=0, \quad j=1, \ldots, n-h
$$

and the matrix $M_{1}^{\perp}$ with entries

$$
\left\langle T_{\tau_{i}^{\perp}} \phi_{\omega}^{0}, E_{1}^{\prime \prime}\left(\phi_{\omega}^{0}\right) T_{\tau_{j}^{\perp}} \phi_{\omega}^{0}\right\rangle, \quad i, j=1, \ldots, n-h
$$

is invertible. Since $E_{1}$ is invariant under $H$, the symmetric bilinear form $E_{1}^{\prime \prime}\left(\phi_{\omega}^{0}\right)$ restricted to the tangent space of the group orbit $G \phi_{\omega}^{0}$ is represented by the symmetric matrix

$$
M_{1}=\left(\begin{array}{cc}
0 & 0 \\
0 & M_{1}^{\perp}
\end{array}\right): \mathfrak{h} \times \mathfrak{h}^{\perp} \longrightarrow \mathfrak{h} \times \mathfrak{h}^{\perp} .
$$

We denote by

$$
\begin{aligned}
\mathcal{L}_{\omega}(\epsilon) & =E_{0}^{\prime \prime}\left(\phi_{\omega}(\epsilon)\right)+\epsilon E_{1}^{\prime \prime}\left(\phi_{\omega}(\epsilon)\right)-Q_{\omega}^{\prime \prime} \\
& =\left[E_{0}^{\prime \prime}\left(\phi_{\omega}^{0}\right)-J^{-1} T_{\omega}\right]+\epsilon\left[E_{0}^{\prime \prime \prime}\left(\phi_{\omega}^{0}\right)\left[\partial_{\epsilon} \phi_{\omega}^{0}\right]+E_{1}^{\prime \prime}\left(\phi_{\omega}^{0}\right)\right]+\mathrm{O}\left(\epsilon^{2}\right)=: \mathcal{L}_{0}+\epsilon \mathcal{L}_{1}+\mathrm{O}\left(\epsilon^{2}\right)
\end{aligned}
$$

the Hessian of the energy $E_{0}+\epsilon E_{1}-Q_{\omega}$ evaluated at the relative equilibrium $\phi_{\omega}(\epsilon)$.

For $\epsilon>0$ the relevant symmetry group is $H$. Thus, the relevant matrix $D$ which we used in $\S 3$ will now be denoted by $D_{H} \in \mathbb{R}^{h \times h}$. The entries of the symmetric matrix $D_{H}$ are given by

$$
\left\langle\partial_{\tau_{i}} \phi_{\omega}(\epsilon), \mathcal{L}_{\omega}(\epsilon) \partial_{\tau_{j}} \phi_{\omega}(\epsilon)\right\rangle=\left\langle\partial_{\tau_{i}} \phi_{\omega}^{0}, \mathcal{L}_{0} \partial_{\tau_{j}} \phi_{\omega}^{0}\right\rangle+\mathrm{O}(\epsilon), \quad i, j=1, \ldots, h .
$$

\subsection{Location of small eigenvalues}

Since we assumed that $D_{G}$ is invertible, $J \mathcal{L}_{\omega}(\epsilon)$ will have $2 n$ small eigenvalues near $\lambda=0$ for $0<\epsilon \ll 1$. We now locate these eigenvalues and determine their Krein signature.

Theorem 4.4 Assume that the hypotheses in Proposition 4.2 are satisfied.

(i) The $2 n$ eigenvalues of $J \mathcal{L}_{\omega}(\epsilon)$ near $\lambda=0$ and the associated eigenvectors are given by

$$
\lambda=\sqrt{\epsilon} \lambda_{1}+\mathrm{O}(\epsilon), \quad u=\sum_{i=1}^{n}\left[T_{\sigma_{i}} \phi_{\omega}^{0}+\sqrt{\epsilon} \lambda_{1} \partial_{\sigma_{i}} \phi_{\omega}^{0}\right] v_{i}+\mathrm{O}(\epsilon)
$$

where $\lambda_{1}$ and $v \in \mathbb{C}^{n}$ satisfy the reduced generalized eigenvalue problem

$$
\left[M_{1}+\lambda_{1}^{2} D_{G}\right] v=0
$$

Due to (4.2), i.e. the equivariance under $H$, there will be at least $2 h$ eigenvalues $\lambda=0$.

(ii) The Krein signature of a simple, non-zero, purely imaginary eigenvalue $\lambda=\sqrt{\epsilon} \lambda_{1}+\mathrm{O}(\epsilon)$ is given by

$$
\operatorname{sign}\left\langle v, M_{1} v\right\rangle=\operatorname{sign}\left\langle v, D_{G} v\right\rangle
$$

where $v$ satisfies (4.6). 
(iii) If $D_{H}$ is invertible, then

$$
k_{\mathrm{r}}+2 k_{\mathrm{c}}+2 k_{\mathrm{i}}^{-}=\mathrm{n}\left(M_{1}\right)+\mathrm{n}\left(D_{G}\right)-\mathrm{n}\left(D_{H}\right) \geq \mathrm{n}\left(M_{1}\right), \quad k_{\mathrm{r}} \geq\left|\mathrm{n}\left(D_{G}\right)-\mathrm{n}\left(D_{H}\right)-\mathrm{n}\left(M_{1}\right)\right|,
$$

where the left-hand sides (introduced in Definition 2.4) refer to the $2 n$ eigenvalues near $\lambda=0$ only.

Note that the reduced eigenvalue problem depends only on the unperturbed wave profile $\phi_{\omega}^{0}$. This is in sharp contrast to the situation when dissipative perturbations are added to a Hamiltonian PDE. In this case, knowledge of the perturbed profile $\phi_{\omega}(\epsilon)$ is typically needed to compute the perturbed eigenvalues (see, for instance, $[10, \S 4-5]$ for an example).

Proof. To find the $2 n$ eigenvalues of $J \mathcal{L}_{\omega}(\epsilon)$ near the origin, we consider the generalized eigenvalue problem

$$
\left[\mathcal{L}_{\omega}(\epsilon)-\lambda J^{-1}\right] u=0,
$$

which we solve for $\lambda$ close to zero using a standard Lyapunov-Schmidt reduction (see [4, $\S$ VII.1]). The null space of the self-adjoint operator $\mathcal{L}_{\omega}(0)=\mathcal{L}_{0}$ is given by the space $Z$ defined in $(2.4)$. We denote by $P$ the orthogonal projection of $X$ onto $Z$ so that $1-P$ projects onto the range of $\mathcal{L}_{0}$. We also write $\lambda=\sqrt{\epsilon} \lambda_{1}$ and $u=u_{0}+u_{1} \in Z \oplus Z^{\perp}$. The idea is now to project (4.8) into its $Z$ and $Z^{\perp}$ components. The equation in $Z^{\perp}$ can be solved uniquely for $u_{1}$. Upon substituting the expression for $u_{1}$ into the $Z$-component of (4.8) and using the identity (3.3), it is straightforward, but tedious, to prove that $(\lambda, u)$ satisfies (4.8) if, and only if, $\left(\lambda_{1}, u_{0}\right)$ with $u_{0} \in Z$ satisfies the reduced eigenvalue problem

$$
\left\langle w_{0},\left[\mathcal{L}_{1}-\lambda_{1}^{2} J^{-1} \mathcal{L}_{0}^{-1} J^{-1}+\mathrm{O}(\sqrt{\epsilon})\right] u_{0}\right\rangle=0, \quad \forall w_{0} \in Z
$$

where $\mathcal{L}_{0}$ and $\mathcal{L}_{1}$ have been defined in $(4.3), \mathcal{L}_{0}^{-1}: Z^{\perp} \rightarrow Z^{\perp}$ and where $u$ and $u_{0}$ are related via $u=$ $\left[1+\lambda \mathcal{L}_{0}^{-1} J^{-1}+\mathrm{O}(\epsilon)\right] u_{0}$. Using (3.3) and the fact that $J$ is skew-symmetric, it is then easy to show that the matrix representation of (4.9) with respect to the basis $T_{\sigma_{j}} \phi_{\omega}^{0}$ of $Z$ coincides with (4.6) provided that

$$
\left\langle T_{\sigma} \phi_{\omega}^{0}, \mathcal{L}_{1} T_{\tau} \phi_{\omega}^{0}\right\rangle=\left\langle T_{\sigma} \phi_{\omega}^{0}, E_{1}^{\prime \prime}\left(\phi_{\omega}^{0}\right) T_{\tau} \phi_{\omega}^{0}\right\rangle
$$

for all $\sigma, \tau \in \mathfrak{g}$. To establish (4.10), fix $\sigma, \tau \in \mathfrak{g}$ and note that

$$
\left\langle T_{\sigma} \phi_{\omega}(\epsilon), H_{\omega}^{\prime \prime}\left(\phi_{\omega}(\epsilon)\right) T_{\tau} \phi_{\omega}(\epsilon)\right\rangle \equiv 0
$$

in $\epsilon$ since $H_{\omega}=E_{0}-Q_{\omega}$ is invariant under $G$ (the above expression is the second variation of $H_{\omega}$ restricted to the group orbit $\left.G \phi_{\omega}(\epsilon)\right)$. Taking the derivative of (4.11) at $\epsilon=0$ gives

$$
\left\langle T_{\sigma} \partial_{\epsilon} \phi_{\omega}^{0}, \mathcal{L}_{0} T_{\tau} \phi_{\omega}^{0}\right\rangle+\left\langle T_{\sigma} \phi_{\omega}^{0}, \mathcal{L}_{0} T_{\tau} \partial_{\epsilon} \phi_{\omega}^{0}\right\rangle+\left\langle T_{\sigma} \phi_{\omega}^{0}, E_{0}^{\prime \prime \prime}\left(\phi_{\omega}^{0}\right)\left[\partial_{\epsilon} \phi_{\omega}^{0}\right] T_{\tau} \phi_{\omega}^{0}\right\rangle=0
$$

The first two terms vanish since $T_{\sigma} \phi_{\omega}^{0}$ lies in the null space of $\mathcal{L}_{0}=H_{\omega}^{\prime \prime}\left(\phi_{\omega}^{0}\right)$. This completes the proof of the first part of the theorem.

Using the results recalled in $\S 2.2$ and the expression (4.5), we see that the Krein signature of the simple, non-zero, purely imaginary eigenvalue $\lambda=\mathrm{i} \sqrt{\epsilon} \mu+\mathrm{O}(\epsilon)$ is given by

$$
\begin{aligned}
\left\langle u, \mathcal{L}_{\omega}(\epsilon) u\right\rangle & =\epsilon\left\langle\sum_{j=1}^{n} v_{j} T_{\sigma_{j}} \phi_{\omega}^{0}, \mathcal{L}_{1} \sum_{j=1}^{n} v_{j} T_{\sigma_{j}} \phi_{\omega}^{0}\right\rangle+\epsilon \mu^{2}\left\langle\sum_{j=1}^{n} v_{j} \partial_{\sigma_{j}} \phi_{\omega}^{0}, \mathcal{L}_{0} \sum_{j=1}^{n} v_{j} \partial_{\sigma_{j}} \phi_{\omega}^{0}\right\rangle+\mathrm{O}\left(\epsilon^{3 / 2}\right) \\
& =\epsilon\left\langle v,\left[M_{1}+\mu^{2} D_{G}\right] v\right\rangle+\mathrm{O}\left(\epsilon^{3 / 2}\right)
\end{aligned}
$$

which gives the desired formula upon using the identity (4.6).

The last part of the theorem is a consequence of Theorem 3.3, in the version proved in $\S 3.4$, applied to

$$
J=\left(\begin{array}{rr}
0 & \mathbb{1} \\
-\mathbb{1} & 0
\end{array}\right), \quad \mathcal{L}=\left(\begin{array}{cc}
M_{1} & 0 \\
0 & D_{G}^{-1}
\end{array}\right),
$$

where $H$ is the underlying symmetry group. The estimate for $k_{\mathrm{r}}$ is a consequence of Remark 3.1 and $\S 3.4$. 


\section{Forced symmetry breaking: global count of eigenvalues}

We continue our discussion of forced symmetry breaking. Throughout this section, we shall assume that we are in the setup of $\S 4$. Specifically, we assume that the assumptions of Proposition 4.2 are satisfied and that $D_{H}$ is invertible. The goal of this section is to calculate the number of eigenvalues of $J \mathcal{L}_{\omega}(\epsilon)$ that are unstable or have negative Krein signature for $\epsilon>0$.

\subsection{General setup}

Without any additional structure, we have the following bound for the number of eigenvalues of $J \mathcal{L}_{\omega}(\epsilon)$ that are unstable or have negative Krein signature for $\epsilon>0$.

Corollary 5.1 Assume that the assumptions in Proposition 4.2 are met and that $D_{H}$ defined in (4.4) is invertible, then we have

$$
k_{\mathrm{r}}+2 k_{\mathrm{c}}+2 k_{\mathrm{i}}^{-} \leq \mathrm{n}\left(\mathcal{L}_{0}\right)+\mathrm{n}\left(M_{1}\right)-\mathrm{n}\left(D_{H}\right)
$$

for $0<\epsilon \ll 1$ where $\mathcal{L}_{0}$ and $M_{1}$ are defined in (4.3) and (4.2), respectively.

Proof. The corollary is a consequence of the counts provided in Theorem 3.2, applied to $J \mathcal{L}_{\omega}(\epsilon)$ with $\epsilon>0$, and Theorem 4.4(iii).

\subsection{Canonical symplectic structure}

Next, we discuss the situation where Hypothesis 2.3 is satisfied for $E=E_{0}+\epsilon E_{1}$. In this case, Theorem 3.3 provides an exact count for eigenvalues of $J \mathcal{L}_{\omega}(\epsilon)$ that are unstable or have negative Krein signature and that are bounded away from the origin uniformly for $0 \leq \epsilon \ll 1$. On the other hand, Theorem 4.4(iii) provides such a count for the $2 n$ eigenvalues of $J \mathcal{L}_{\omega}(\epsilon)$ that are close to the origin $0<\epsilon \ll 1$. Combining these counts gives the following corollary.

Corollary 5.2 If the assumptions in Proposition 4.2 and Hypothesis 2.3 are met and if $D_{H}$ defined in (4.4) is invertible, then we have

$$
k_{\mathrm{r}}+2 k_{\mathrm{c}}+2 k_{\mathrm{i}}^{-}=\mathrm{n}\left(\mathcal{L}_{0}\right)+\mathrm{n}\left(M_{1}\right)-\mathrm{n}\left(D_{H}\right)
$$

for $0<\epsilon \ll 1$ where $\mathcal{L}_{0}$ and $M_{1}$ are defined in (4.3) and (4.2), respectively.

\subsection{Unperturbed constrained minimizers}

On account of [8, Theorem 4.1], the unperturbed wave is nonlinearly stable for $\epsilon=0$ provided $\mathrm{n}\left(\mathcal{L}_{0}\right)=\mathrm{n}\left(D_{G}\right)$. In this situation, there are therefore no unstable eigenvalues or eigenvalues with negative Krein signature for the unperturbed problem. Thus, the $2 n$ eigenvalues of $J \mathcal{L}_{\omega}(\epsilon)$ found in Theorem 4.4(iii) account for all eigenvalues that are unstable or have negative Krein signature for $0<\epsilon \ll 1$.

Corollary 5.3 Assume that the assumptions in Proposition 4.2 are met, that $D_{H}$ defined in (4.4) is invertible, and that $\mathrm{n}\left(\mathcal{L}_{0}\right)=\mathrm{n}\left(D_{G}\right)$. We then have

$$
k_{\mathrm{r}}+2 k_{\mathrm{c}}+2 k_{\mathrm{i}}^{-}=\mathrm{n}\left(M_{1}\right)+\mathrm{n}\left(D_{G}\right)-\mathrm{n}\left(D_{H}\right), \quad k_{\mathrm{r}} \geq\left|\mathrm{n}\left(D_{G}\right)-\mathrm{n}\left(D_{H}\right)-\mathrm{n}\left(M_{1}\right)\right|
$$

for $0<\epsilon \ll 1$. 


\subsection{Edge bifurcation eigenvalues}

Lastly, we address the existence of eigenvalues that emerge from the essential spectrum - in other words, the associated eigenfunctions do not have well-defined limits in the underlying function space as $\epsilon \rightarrow 0$. We refer to such bifurcations as edge bifurcations since they can, at least for integrable PDEs, occur only at branch points of the linear dispersion relation, with one of them being located at the edge of the essential spectrum [12]. Of interest here is the question whether edge bifurcation can lead to eigenvalues that are unstable or have negative Krein signature.

First suppose that Hypothesis 2.3 is satisfied. In this case, Theorems 3.3 and 4.4 and Corollary 5.2 show that all unstable eigenvalues as well as eigenvalues with negative Krein signature are small perturbations of the eigenvalues at $\epsilon=0$. Hence, any eigenvalues arising from an edge bifurcation will be purely imaginary and have positive Krein signature. Using Theorems 3.3 and 4.4 and Corollary 5.3 shows that the same conclusion applies to problems where the unperturbed wave is a constrained minimizer. Thus, we obtain the following result:

Corollary 5.4 Assume that the hypotheses in Proposition 4.2 are met and that $D_{H}$, defined in (4.4), is invertible. If Hypothesis 2.3 is met, or if $\mathrm{n}\left(\mathcal{L}_{0}\right)=\mathrm{n}\left(D_{G}\right)$, then any eigenvalues arising from an edge bifurcation will be purely imaginary with positive Krein signature.

We do not know whether Corollary 5.4 is true when Hypothesis 2.3 is not met and the unperturbed wave is not a constrained minimizer. In this situation, the assertion of Corollary 5.4 could potentially fail when, as $\epsilon$ crosses through zero, a pair of resonance poles for $\epsilon<0$ becomes a pair of unstable eigenvalues for $\epsilon>0$. At $\epsilon=0$, the resonance pole would be embedded in the essential spectrum, for instance at a branch point, but would not correspond to an eigenvalue, i.e., the associated eigenfunction would not be contained in the underlying function space. Such resonance poles arise, and can be tracked, as roots of the Evans function $[11,16]$. Unfortunately, we do not know of any examples where this scenario occurs. It was proved in [16], however, that for the generalized KdV equation, which does not fit in the framework of this article as $J$ is not bijective, that a resonance pole goes through the origin and creates a positive real eigenvalue as a parameter is varied.

\section{$6 \quad$ Weakly coupled nonlinear Schrödinger equations}

We now apply the results obtained in the previous section to coupled nonlinear Schrödinger equations. Rather than presenting the most general setup possible, we will focus instead on a single representative example. We emphasize, however, that Theorems 3.3 and 4.4 are applicable to a quite general class of coupled NLS equations.

\subsection{Formulation}

Consider the system

$$
\partial_{t} q_{j}=\mathrm{i}\left[\frac{1}{2} \partial_{x x} q_{j}+\left|q_{j}\right|^{2} q_{j}\right], \quad x \in \mathbb{R}, \quad j=1, \ldots, N
$$

of $N$ uncoupled nonlinear Schrödinger equations. Writing $q_{j}=u_{j}+\mathrm{i} v_{j}$, we see that (6.1) can be written as

$$
\partial_{t}\left(\begin{array}{c}
u_{j} \\
v_{j}
\end{array}\right)=\left(\begin{array}{rr}
0 & \mathbb{1} \\
-\mathbb{1} & 0
\end{array}\right)\left(\begin{array}{l}
-\frac{1}{2} \partial_{x x} u_{j}-\left(u_{j}^{2}+v_{j}^{2}\right) u_{j} \\
-\frac{1}{2} \partial_{x x} v_{j}-\left(u_{j}^{2}+v_{j}^{2}\right) v_{j}
\end{array}\right), \quad j=1, \ldots, N
$$


which is Hamiltonian with energy

$$
E_{0}(u, v)=\frac{1}{2} \sum_{j=1}^{N} \int_{-\infty}^{\infty}\left[\left(\partial_{x} u_{j}\right)^{2}+\left(\partial_{x} v_{j}\right)^{2}-\left(u_{j}^{2}+v_{j}^{2}\right)^{2}\right] \mathrm{d} x .
$$

We work in the Hilbert spaces

$$
U=H^{2}\left(\mathbb{R}, \mathbb{R}^{N}\right) \times H^{2}\left(\mathbb{R}, \mathbb{R}^{N}\right), \quad X=H^{1}\left(\mathbb{R}, \mathbb{R}^{N}\right) \times H^{1}\left(\mathbb{R}, \mathbb{R}^{N}\right), \quad V=L^{2}\left(\mathbb{R}, \mathbb{R}^{N}\right) \times L^{2}\left(\mathbb{R}, \mathbb{R}^{N}\right)
$$

and shall often identify $H^{k}\left(\mathbb{R}, \mathbb{R}^{N}\right) \times H^{k}\left(\mathbb{R}, \mathbb{R}^{N}\right)$ with $H^{k}\left(\mathbb{R}, \mathbb{C}^{N}\right)$ via $q=u+\mathrm{i} v$. Equation (6.1) is invariant under the Lie group $G=\mathbb{R}^{N} \times \mathbb{T}^{N}$, where $\mathbb{T}=S^{1}$, which acts on $V$ according to

$$
[T(g) q]_{j}(x)=\mathrm{e}^{\mathrm{i} \theta_{j}} q_{j}\left(x+\xi_{j}\right), \quad g=(\xi, \theta) \in G=\mathbb{R}^{N} \times \mathbb{T}^{N}
$$

so that the generators are, in complex notation, given by

$$
T_{\left(e_{j}, 0\right)} q=\partial_{x} q_{j} e_{j}, \quad T_{\left(0, e_{j}\right)} q=\mathrm{i} q_{j} e_{j}, \quad j=1, \ldots, N
$$

where $e_{j} \in \mathbb{R}^{N}$ is the $j$ th canonical basis vector. Relative equilibria $\phi_{(c, \alpha)} \in U$ of (6.1) with $(c, \alpha) \in$ $\mathbb{R}^{N} \times \mathbb{R}^{N}=\mathfrak{g}$ are given by

$$
\phi_{(c, \alpha)}(x)=\left(\mathrm{e}^{-\mathrm{i} c_{1} x} \Phi_{\alpha_{1}-c_{1}^{2} / 2}(x), \ldots, \mathrm{e}^{-\mathrm{i} c_{N} x} \Phi_{\alpha_{N}-c_{N}^{2} / 2}(x)\right) \in \mathbb{C}^{N}
$$

where $\Phi_{\omega}(x)=\sqrt{2 \omega} \operatorname{sech}(\sqrt{2 \omega} x)$ for $\omega>0$. The time evolution of $\phi_{(c, \alpha)}$ is simply $T(c t, \alpha t) \phi_{(c, \alpha)}$. We shall focus on the wave

$$
\phi_{\omega}(x):=\left(\Phi_{\omega}(x), \ldots, \Phi_{\omega}(x), 0, \ldots, 0\right)
$$

that is obtained by choosing $c=0$ and $\alpha_{j}=\omega>0$ for all $j$. Using (6.3), it is now easy to see that

$$
T_{\left(e_{j}, 0\right)} \phi_{\omega}=\partial_{x} \Phi_{\omega} e_{j}, \quad T_{\left(0, e_{j}\right)} \phi_{\omega}=\Phi_{\omega} e_{j+N}, \quad \partial_{\left(e_{j}, 0\right)} \phi_{\omega}=-x \Phi_{\omega} e_{j+N}, \quad \partial_{\left(0, e_{j}\right)} \phi_{\omega}=\partial_{\omega} \Phi_{\omega} e_{j} .
$$

In particular, we obtain

$$
D_{G}=\left(\begin{array}{cc}
\left\langle\partial_{x} \Phi_{\omega},-x \Phi_{\omega}\right\rangle_{L^{2}} \mathbb{1}_{N} & 0 \\
0 & -\left\langle\Phi_{\omega}, \partial_{\omega} \Phi_{\omega}\right\rangle_{L^{2}} \mathbb{1}_{N}
\end{array}\right)=\left(\begin{array}{cc}
\sqrt{2 \omega} \mathbb{1}_{N} & 0 \\
0 & -\sqrt{2 \omega}^{-1} \mathbb{1}_{N}
\end{array}\right)
$$

and therefore $\mathrm{n}\left(D_{G}\right)=N$. Note that the upper-left and lower-right blocks of $D_{G}$ corresponds to translation symmetry and phase invariance, respectively. The linearization of (6.2) about the wave $\phi_{\omega}$ is given by $J \mathcal{L}_{\omega}$ where

$$
J=\left(\begin{array}{cc}
0 & \mathbb{1} \\
-\mathbb{1} & 0
\end{array}\right), \quad \mathcal{L}_{\omega}=\left(\begin{array}{cc}
L_{+} & 0 \\
0 & L_{-}
\end{array}\right)
$$

with $L_{+}=\operatorname{diag}\left(L_{\mathrm{r}}, \ldots, L_{\mathrm{r}}\right), L_{-}=\operatorname{diag}\left(L_{\mathrm{i}}, \ldots, L_{\mathrm{i}}\right)$ and

$$
L_{\mathrm{r}}=-\frac{1}{2} \partial_{x x}+\omega-3 \Phi_{\omega}^{2}(x), \quad L_{\mathrm{i}}=-\frac{1}{2} \partial_{x x}+\omega-\Phi_{\omega}^{2}(x) .
$$

In particular, Hypotheses 2.1, 2.2 and 2.3 are satisfied. Furthermore, by Sturm-Liouville theory, we have $\mathrm{n}\left(L_{+}\right)=N$ and $\mathrm{n}\left(L_{-}\right)=0$ so that $\mathrm{n}\left(\mathcal{L}_{\omega}\right)=N$. Thus, on account of Theorem 3.1, the unperturbed wave is a constrained minimizer.

\subsection{Symmetry-breaking perturbations}

We now add a small perturbation $\epsilon E_{1}(u, v)$ to the energy $E_{0}$ and consider the perturbed NLS equation

$$
\partial_{t}\left(\begin{array}{l}
u \\
v
\end{array}\right)=\left(\begin{array}{rr}
0 & \mathbb{1} \\
-\mathbb{1} & 0
\end{array}\right)\left(E_{0}+\epsilon E_{1}\right)^{\prime}(u, v)
$$


where we assume that $E_{1}: X \rightarrow \mathbb{R}$ is smooth and invariant under a subgroup $H$ of $G$. Prototypical examples are

$$
E_{1}^{\prime}(u, v)=f\left(|u|^{2}+|v|^{2}\right)\left(\begin{array}{c}
u \\
v
\end{array}\right), \quad H=\mathbb{R} \times \mathbb{T}^{N}=\mathbb{R} e \times \mathbb{T}^{N} \leq G=\mathbb{R}^{N} \times \mathbb{T}^{N},
$$

and

$$
E_{1}^{\prime}(u, v)=\left(\begin{array}{c}
C u \\
C v
\end{array}\right), \quad H=\mathbb{R} \times \mathbb{R}=\mathbb{R} e \times \mathbb{R} e \leq G=\mathbb{R}^{N} \times \mathbb{T}^{N}
$$

where $C \in \mathbb{R}^{N \times N}$ is a symmetric coupling matrix and $e=(1, \ldots, 1) \in \mathbb{R}^{N}$. Thus, in (6.7), all rotational symmetries and simultaneous translations in $x$ in all components are preserved ${ }^{2}$, while in (6.8) only simultaneous translations and rotations are preserved.

We shall assume that the wave $\phi_{\omega}$ persists as a smooth function $\phi_{\omega}(\epsilon)$ for $\epsilon>0$ and refer to Proposition 4.2 for sufficient conditions for this to occur. We are then interested in the fate of the $4 N$ small eigenvalues of the linearization $J \mathcal{L}_{\omega}(\epsilon)$, calculated in the co-rotating frame $(u, v) \mapsto \mathrm{e}^{\mathrm{i} \omega t}(u, v)$, of (6.6) about the wave $\phi_{\omega}(\epsilon)$. We emphasize that the results in $\S 4$ depend only on the unperturbed profile $\phi_{\omega}$ given in (6.4).

Note first that the symmetry $H$ will tie $h$ small eigenvalues, each with algebraic multiplicity at least two, to the origin. In fact, the algebraic multiplicity will be exactly two if, and only if, the matrix $D_{H}$, defined in (4.4), is invertible. The matrix $D_{H}$ can be calculated as follows. We observe from (4.4) that $D_{H}$ is, to leading order in $\epsilon$, the restriction of the bilinear form $D_{G}$ to $\mathfrak{h} \times \mathfrak{h}$. Thus, we identify $\mathfrak{g}=\mathbb{R}^{2 N}$, choose orthonormal bases $\tau_{j} \in \mathfrak{h} \subset \mathbb{R}^{2 N}, j=1, \ldots, h$, of $\mathfrak{h}$ and $\tau_{j}^{\perp} \in \mathfrak{h}^{\perp} \subset \mathbb{R}^{2 N}, j=1, \ldots, 2 N-h$, of $\mathfrak{h}^{\perp}$, and define the matrices $T_{\mathfrak{h}} \in \mathbb{R}^{2 N \times h}$ with columns $\tau_{j}$ and $T_{\mathfrak{h}} \perp \in \mathbb{R}^{2 N \times(2 N-h)}$ with columns $\tau_{j}^{\perp}$. We then have

$$
D_{H}=T^{t} D_{G} T \in \mathbb{R}^{h \times h} .
$$

For instance, the matrices associated with the symmetry groups $H$ in (6.7) and (6.8) are invertible.

To apply Theorem 4.4, we also need the symmetric matrix $M_{1} \in \mathbb{R}^{2 N \times 2 N}$ which is given by

$$
M_{1}=\left(\begin{array}{cc}
\left(\left\langle\partial_{x} \Phi_{\omega} e_{i}, E_{1}^{\prime \prime}\left(\phi_{\omega}\right) \partial_{x} \Phi_{\omega} e_{j}\right\rangle_{L^{2}}\right)_{i j} & \left(\left\langle\partial_{x} \Phi_{\omega} e_{i}, E_{1}^{\prime \prime}\left(\phi_{\omega}\right) \Phi_{\omega} e_{j+N}\right\rangle_{L^{2}}\right)_{i j} \\
\left(\left\langle\Phi_{\omega} e_{i+N}, E_{1}^{\prime \prime}\left(\phi_{\omega}\right) \partial_{x} \Phi_{\omega} e_{j}\right\rangle_{L^{2}}\right)_{i j} & \left(\left\langle\Phi_{\omega} e_{i+N}, E_{1}^{\prime \prime}\left(\phi_{\omega}\right) \Phi_{\omega} e_{j+N}\right\rangle_{L^{2}}\right)_{i j}
\end{array}\right), \quad M_{1}^{\perp}=T_{\mathfrak{h} \perp}^{t} M_{1} T_{\mathfrak{h} \perp}
$$

where $i, j=1, \ldots, N$ so that each block matrix in the above expression is in $\mathbb{R}^{N \times N}$. An application of Theorem 4.4 gives the following result.

Proposition 6.1 Assume that the matrices $D_{H}$ and $M_{1}^{\perp}$, defined in (4.4) and (6.10), are invertible.

(i) The $4 N$ eigenvalues of $J \mathcal{L}_{\omega}(\epsilon)$ near the origin are given by $\lambda=\sqrt{\epsilon} \lambda_{1}+\mathrm{O}(\epsilon)$ where $\lambda_{1}$ satisfies

$$
\left[M_{1}+\lambda_{1}^{2} D_{G}\right] v=0
$$

for some $v \in \mathbb{C}^{2 N}$. There are at least $2 h$ eigenvalues $\lambda$ at zero.

(ii) The Krein signature of a simple, non-zero, purely imaginary eigenvalue $\lambda=\sqrt{\epsilon} \lambda_{1}+\mathrm{O}(\epsilon)$ is given by $\operatorname{sign}\left(2 \omega\left|v_{1}\right|^{2}-\left|v_{2}\right|^{2}\right)$ where $v=\left(v_{1}, v_{2}\right) \in \mathbb{R}^{N} \times \mathbb{R}^{N}$ satisfies (6.11).

(iii) We have

$$
k_{\mathrm{r}}+2 k_{\mathrm{c}}+2 k_{\mathrm{i}}^{-}=N+\mathrm{n}\left(M_{1}\right)-\mathrm{n}\left(D_{H}\right), \quad k_{\mathrm{r}} \geq\left|N-\mathrm{n}\left(D_{H}\right)-\mathrm{n}\left(M_{1}\right)\right|
$$

where the left-hand sides have been introduced in Definition 2.4. All of these eigenvalues have modulus of order $\mathrm{O}(\sqrt{\epsilon})$ and are therefore captured by (6.11).

(iv) Eigenvalues arising through edge bifurcations at $\lambda= \pm \mathrm{i} \omega$ are purely imaginary with positive Krein signature.

\footnotetext{
${ }^{2}$ We remark that the results in [17] are not applicable to (6.6) since the Evans function vanishes at the branchpoint $\lambda= \pm \mathrm{i} \omega$ for $\epsilon=0$ (see [11])
} 


\subsection{Reversible perturbations}

Next, we consider the important situation when $E_{1}$ is reversible. Thus, we shall assume that the following assumption is met.

Hypothesis 6.2 Assume that $E_{1}: X \rightarrow \mathbb{R}$ is invariant under the $\mathbb{Z}_{2}$-symmetry induced on $X$ by the bounded operator $\mathcal{R}: L^{2}(\mathbb{R}, \mathbb{R}) \rightarrow L^{2}(\mathbb{R}, \mathbb{R})$ with $[\mathcal{R} u](x)=u(-x)$ for $x \in \mathbb{R}$.

Since $\Phi_{\omega}(x)$ is even in $x$, we see that $E_{1}^{\prime \prime}\left(\phi_{\omega}\right)$ commutes with $\mathcal{R}$ and therefore maps even functions into even functions and odd functions into odd functions. In fact, the same statement is true for the full linearization $J \mathcal{L}_{\omega}(\epsilon)$ about the perturbed wave. Thus, $J \mathcal{L}_{\omega}(\epsilon)$ maps both $\operatorname{Fix}(\mathrm{R})$ and $\operatorname{Fix}(-\mathrm{R})$ into themselves for all $0 \leq \epsilon \ll 1$, so that even and odd eigenfunctions (and the associated eigenvalues) do not interact with each other. As a consequence, we see that $M_{1}$ is, in fact, of block-diagonal form:

$$
M_{1}=\left(\begin{array}{cc}
M_{\mathrm{trans}} & 0 \\
0 & M_{\mathrm{rot}}
\end{array}\right)
$$

where both $M_{\text {trans }}$ and $M_{\text {rot }}$ are symmetric. In particular, the reduced eigenvalue problem (6.11) decouples:

$$
\left[M_{\text {trans }}+\lambda_{1}^{2} \sqrt{2 \omega} \mathbb{1}_{N}\right] v_{1}=0, \quad\left[M_{\text {rot }}-\lambda_{1}^{2} \sqrt{2 \omega}^{-1} \mathbb{1}_{N}\right] v_{2}=0
$$

If the matrices $D_{H}$ and $M_{1}^{\perp}$ are invertible, we can apply Proposition 6.1(ii) and Remark 3.1 separately to the eigenvalue problem posed on odd functions and on even functions. In the odd case, we see that $M_{\text {trans }}$ yields $\mathrm{p}\left(M_{\text {trans }}\right)$ pairs of non-zero, purely imaginary eigenvalues with necessarily positive Krein signature and $\mathrm{n}\left(M_{\text {trans }}\right)$ unstable real eigenvalues. In the even case, we obtain $\mathrm{p}\left(M_{\text {rot }}\right)$ unstable real eigenvalues and $\mathrm{n}\left(M_{\text {rot }}\right)$ pairs of non-zero, purely imaginary eigenvalues with necessarily negative Krein signature. Formulating this statement slightly differently and using the remark after Hypothesis 6.2 which shows that the decomposition (6.13) is preserved for the full linearization, we obtain the following proposition.

Proposition 6.3 If the matrices $D_{H}$ and $M_{1}^{\perp}$ are invertible, and Hypothesis 6.2 is met, then

$$
k_{\mathrm{r}}=\mathrm{p}\left(M_{\text {rot }}\right)+\mathrm{n}\left(M_{\text {trans }}\right), \quad k_{\mathrm{i}}^{-}=\mathrm{n}\left(M_{\text {rot }}\right), \quad k_{\mathrm{c}}=0 .
$$

\subsection{Dissipative perturbations}

Lastly, we comment briefly on the system

$$
u_{t}=[J-\epsilon \mathbb{1}] E^{\prime}(u), \quad 0 \leq \epsilon \ll 1
$$

where the Hamiltonian structure (but not the equivariance under $G$ ) is broken by the particular dissipative perturbation $E^{\prime}(u)$, i.e., the energy itself. In this case, any relative equilibrium $\phi_{\omega}$ persists with no change of its profile. Furthermore, the linearization of (6.14) about $\phi_{\omega}$ is given by $\mathcal{A}(\epsilon)=[J-\epsilon \mathbb{1}] \mathcal{L}_{\omega}$.

It is not hard to show, see [6, p. 305] and [14], that a non-zero simple pair of purely imaginary eigenvalues of $\mathcal{A}$ moves, for $\epsilon>0$, into the unstable right half-plane if it has negative Krein signature and into the left halfplane if it has positive Krein signature. We discuss here the effect that adding the dissipative perturbation (6.14) has on the eigenvalues at the origin that occur in Jordan blocks. Note that we have

$$
\begin{aligned}
\mathcal{A}(\epsilon) & =[J-\epsilon \mathbb{1}] \mathcal{L}_{\omega}, & \mathcal{A}_{0} & =\mathcal{A}(0)=J \mathcal{L}_{\omega}, & \mathcal{A}_{0}^{\prime} & =-\mathcal{L}_{\omega} \\
u_{0} & =T_{\sigma} \phi_{\omega}, & w_{0} & =\partial_{\sigma} \phi_{\omega}, & w_{0}^{*} & =J^{-1} u_{0}=\mathcal{L}_{\omega} w_{0} \\
\mathcal{A}(\epsilon) u_{0} & =0, & \mathcal{A}_{0} w_{0} & =u_{0}, & \mathcal{A}_{0}^{*} w_{0}^{*} & =0
\end{aligned}
$$


where we used (3.2) and (3.3). Thus, the symmetry group $G$ still enforces the eigenvalue $\lambda=0$ with eigenfunction $u_{0}=T_{\sigma} \phi_{\omega}$. On account of $[10,(4.5)]$, the other eigenvalue of the Jordan block is given by

$$
\lambda(\epsilon)=\frac{\left\langle w_{0}^{*}, \mathcal{A}_{0}^{\prime} w_{0}-u_{0}^{\prime}\right\rangle}{\left\langle w_{0}^{*}, w_{0}\right\rangle} \epsilon+\mathrm{O}\left(\epsilon^{2}\right) .
$$

Substituting the relevant expressions from (6.15), we obtain

$$
\lambda(\epsilon)=-\frac{\left|\mathcal{L}_{\omega} \partial_{\sigma} \phi_{\omega}\right|^{2}}{\left\langle\partial_{\sigma} \phi_{\omega}, \mathcal{L}_{\omega} \partial_{\sigma} \phi_{\omega}\right\rangle} \epsilon+\mathrm{O}\left(\epsilon^{2}\right)
$$

Note that the matrix $D_{G}$ is formed exactly by the scalar products in the denominator. Thus, if we now specialize to the nonlinear Schrödinger equation (6.1), we infer from (6.5) that translational eigenvalues will move into the left half-plane, while rotational eigenvalues will move into the unstable right half-plane.

We may therefore expect that dissipative perturbations will stabilize the translational eigenvalues, while the eigenvalues arising from the phase invariance are amenable to a destabilization. This is indeed what has been observed in the perturbation from the NLS to complex Ginzburg-Landau equations. This observation also indicates that adding energy in the direction of eigenfunctions that correspond to broken phase invariances may help to stabilize waves.

\section{Discussion}

\section{Non-abelian symmetry groups}

Our abstract results in $\S 3$ are applicable to Hamiltonian PDEs that are equivariant under the action of an abelian Lie group $G$ and to relative equilibria with trivial isotropy. Specifically, our results are therefore applicable to radially-symmetric waves of nonlinear Schrödinger equations posed on $\mathbb{R}^{d}$ with $d \geq 2$. They do, however, not apply to non-radially symmetric rotating waves (spiral waves or vortices) on $\mathbb{R}^{d}$ since $G$ will then contain the Euclidean symmetry group $\operatorname{SE}(d)$ of $\mathbb{R}^{d}$ which is not abelian. The issue is that we have

$$
\mathcal{L}_{\omega} T_{\sigma} \phi_{\omega}=T_{[\sigma, \omega]} \phi_{\omega}
$$

so that non-abelian Lie groups enforce not only spectrum at the origin but also potentially on the imaginary axis away from zero (see, for instance, [18] for a discussion). We believe that it is possible to extend the results presented here to cover non-abelian Lie groups.

\section{Krein signature and the Evans function}

Theorem 3.3 provides an exact count of eigenvalues that are either unstable or have negative Krein signature. It would be desirable to locate these eigenvalues, specifically the purely imaginary eigenvalues with negative Krein signature (since the number of unstable eigenvalues can then be computed using the count provided in Theorem 3.3). One possible tool for locating and tracking eigenvalues is the Evans function [1, 11, 16]. In light of the above discussion, it would be interesting to see how the Krein signature of an eigenvalue is encoded in the Evans function. Given the fact that only eigenvalues of opposite signature can leave the imaginary axis upon collision, while eigenvalues of identical signature are confined to the imaginary axis, the Krein signature must be encoded somehow in the Evans function but, alas, we do not know in which way.

\section{References}

[1] J. Alexander, R. A. Gardner and C. K. R. T. Jones. A topological invariant arising in the stability of travelling waves. J. Reine Angew. Math. 410 (1990) 167-212. 
[2] S. Cuccagna, D. Pelinovsky and V. Vougalter. Spectra of positive and negative energies in the linearized NLS problem. Preprint (September 2003).

[3] M. Dellnitz, I. Melbourne and J. E. Marsden. Generic bifurcation of Hamiltonian vector fields with symmetry. Nonlinearity 5 (1992) 979-996.

[4] M. Golubitsky and D. Schaeffer. Singularities and Groups in Bifurcation Theory, Vol. 1. Springer-Verlag, 1985.

[5] M. Grillakis. Linearized instability for nonlinear Schrödinger and Klein-Gordon equations. Comm. Pure Appl. Math. 46 (1988) 747-774.

[6] M. Grillakis. Analysis of the linearization around a critical point of an infinite dimensional Hamiltonian system. Comm. Pure Appl. Math. 43 (1990) 299-333.

[7] M. Grillakis, J. Shatah and W. Strauss. Stability theory of solitary waves in the presence of symmetry I. J. Funct. Anal. 74 (1987) 160-197.

[8] M. Grillakis, J. Shatah and W. Strauss. Stability theory of solitary waves in the presence of symmetry II. J. Funct. Anal. 94 (1990) 308-348.

[9] T. Kapitula. Stability of waves in perturbed Hamiltonian systems. Physica D 156 (2001) 186-200.

[10] T. Kapitula, J. N. Kutz and B. Sandstede. The Evans function for nonlocal equations. Indiana Univ. Math. J. (accepted).

[11] T. Kapitula and B. Sandstede. Stability of bright solitary wave solutions to perturbed nonlinear Schrödinger equations. Physica D 124 (1998) 58-103.

[12] T. Kapitula and B. Sandstede. Edge bifurcations for near integrable systems via Evans function techniques. SIAM J. Math. Anal. 33 (2002) 1117-1143.

[13] R. S. MacKay. Stability of equilibria of Hamiltonian systems. In: Hamiltonian Dynamical Systems (R. S. MacKay and J. Meiss, eds.). Adam Hilger, 1987, 137-153.

[14] R. S. MacKay. Movement of eigenvalues of Hamiltonian equilibria under non-Hamiltonian perturbation. Phys. Lett. A 155 (1991) 266-268.

[15] J. H. Maddocks. Restricted quadratic forms and their application to bifurcation and stability in constrained variational principles. SIAM J. Math. Anal. 16 (1985) 47-68. [Errata: SIAM J. Math. Anal. 19 (1988) 1256$1257]$.

[16] R. Pego and M. Weinstein. Eigenvalues, and instabilities of solitary waves. Phil. Trans. R. Soc. Lond. A 340 (1992) 47-94.

[17] D. Pelinovsky. Inertia law for spectral stability of solitary waves in coupled nonlinear Schrödinger equations. Preprint (September 2003).

[18] B. Sandstede, A. Scheel and C. Wulff. Bifurcations and dynamics of spiral waves. J. Nonlinear Sci. 9 (1999) 439-478.

[19] D. Skryabin. Energy of internal modes of nonlinear waves and complex frequencies due to symmetry breaking. Phys. Rev. E 64 (2001) 055601. 